\title{
Determination of the Critical Wind Speed and Stability of the Bundled Phase at Galloping Overhead Lines
}

\author{
Muratkali Dzhamanbayev ${ }^{1}$ and Nurmakhan Tokenov ${ }^{2}$ \\ ${ }^{1,2}$ Electrical Engineering and Automation of Technological complexes, Kazakh National Technical University named after K.I. \\ Satpayev, Almaty, Kazakhstan
}

\begin{abstract}
This article, based on linearized equation of movement, considers an aerodynamical stability of bundled phase of overhead lines, covered with ice in wind flow. The Hurwitz criterion is used for determining critical speed of wind flow, when appears loss of stability of bundled phase. It is analyzed relationship between critical speed and lines characteristics, wire tension and attenuation ratio. In the end of this article appropriate conclusions are made.
\end{abstract}

Keywords-overhead transmission lines; bundled phase; galloping conductors; stability criterion; critical wind speed

\section{INTRODUCTION}

As is known, maximum powers, which may be transferred through ac transmission line, can be greatly increased not only by means of voltage transfer growth, but also by means of overhead transmission's phase bundling into several wires. It improves electrical characteristics of overhead transmission. Nevertheless, as exploitation experience shows, bundled phase is more exposed to galloping, which leads to reliability decrease of electrical energy transmission. That's why, researches of reactions of overhead transmission lines bundled phase on wind stress in conditions of ice-up and development of galloping control methods are one of the actual up-to-date problems.

Review of previous researches. Research background

Papers [1-5, 7, 9, 12-17] are considered with studying of galloping phenomenon. In these works, basically, considered theoretical problems of galloping, which consist of making general mathematical model, which under given conditions allows to determine main characteristics of oscillatory process - galloping intensity. Besides, in papers [2, 6, 8, 16-18] high attention is paid to methods of galloping control. However, issues connected with iced-up wires stability loss in wind flow are not considered. The problems of aero-elastic instability of elastically fixed plate and determination of aerodynamical flatter critical speed and divergence are considered in paper [9, $16,17]$. However, elastically fixed plate inadequately describes overhead transmission lines behavior. Among the recent work considered determination of overhead transmission lines critical wind speed galloping conductors can be emphasized the paper $[3,6-8]$. In this work considered a single wire. This paper considers the stability problems of bundled phase of overhead transmission lines, and this is a distinctive feature of this work. Problems of bundled phase stability

\section{PROBLEMS OF BUNDLED PHASE STABILITY}

This research is aimed to determine the wind flow critical speed, which comes with the loss of stability bundled phase, covered with ice in wind flow. Bundled phase is a bunch, which consists of several wires. Figure I shows the most frequent configurations of overhead lines.

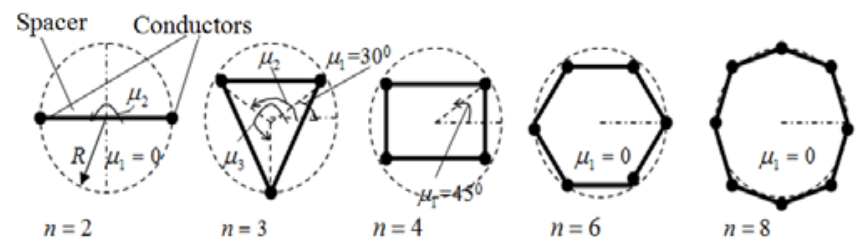

FIGURE I. BUNDLED PHASES' CONFIGURATION. N IS NUMBER OF WIRES IN PHASE (NUMBER OF BUNDLES), R IS BUNDLE RADIUS, MI IS ANGLE, WHICH CHARACTERIZE RELATIVE POSITION OF CERTAIN WIRES IN LOOM.

Paper [10, 11, 17] provides mathematical model of overhead lines galloping with two degrees of freedom. Bundled phases movement, covered with ice, in wind current is described by combined nonlinear differential second-order equation. For the research of aerodynamical instability of overhead lines, nonlinear differential equations are linearized in neighborhood of reference point. Results of linearization are given below

$$
\left\{\begin{array}{l}
\ddot{a}(t)+\left(\frac{\delta_{L} \omega_{L}}{\pi}-k_{1} V\right) \dot{a}(t)+\frac{k_{2}}{V} \dot{a}^{3}(t)+k_{3} V \dot{a}(t) \varphi^{2}(t)-k_{3} \dot{a}^{2}(t) \varphi(t)+\omega_{\Lambda}^{2} a(t)+ \\
+k_{4} a^{2}(t)+k_{5} a^{3}(t)+k_{6} a(t) \varphi^{2}(t)+k_{7} V^{2} \varphi(t)+k_{8} \varphi^{2}(t)-k_{2} V^{2} \varphi^{3}(t)+k_{9} V^{4}=0 \\
\ddot{\varphi}(t)+\frac{\delta_{k} \omega_{L}}{\pi} \dot{\varphi}(t)+\left(s_{1} \omega_{\Lambda}{ }^{2}+k_{10} V^{2}\right) \varphi(t)+\left(k_{11}-k_{12} V^{2}\right) \varphi^{3}(t)-k_{10} V \dot{a}(t)+ \\
+\frac{k_{12}}{V} \dot{a}^{3}(t)+k_{13} V \dot{a}(t) \varphi^{2}(t)-k_{13} \dot{a}^{2}(t) \varphi(t)+k_{14} a(t) \varphi(t)+k_{15} a^{2}(t) \varphi(t)=0
\end{array}\right.
$$

where

$$
\begin{aligned}
& \xi=\xi_{0} V^{2} ; \quad \xi_{0}=\frac{\rho d_{\Gamma} C_{D 0}}{2 P_{B e p}} ; b_{2}=\frac{g \rho d_{\Gamma}}{P_{P}} ; b_{3}=\frac{b_{2} d_{\Gamma}}{R^{2}} ; \\
& k_{10}=\frac{2 b_{3}}{\pi} C_{M 0} ; k_{1}=\frac{2 b_{2}}{\pi}\left(C_{D 0}+C_{L 0}\right) ; k_{7}=\frac{2 b_{2}}{\pi} C_{L 0} ;
\end{aligned}
$$




$$
\begin{aligned}
& k_{9}=b_{2} C_{D 0} \xi_{0} ; \quad s_{1}=\frac{1}{n} \sum_{i=1}^{n} \cos ^{2}\left(\mu_{i}-\xi\right) ; \\
& \omega_{\Lambda}{ }^{2}=\frac{\pi^{2} g T}{P_{P} \ell^{2}}\left(1+\frac{8 E F P_{P}^{2} \ell^{2}}{\pi^{4} T^{3}}\right) .
\end{aligned}
$$

In this case:

$a(t)$ and $\varphi(t)$ are joint coordinates of linear and angular displacement, $\xi$ is angle of bundled phase displacement under wind current stress, $\rho$ is air density, $d_{\Gamma}$ is distinctive size of precipitations cross-section, $\ell$ is length of spans, $T$ is tension of displaced wire, $E$ is Young's modulus, $F$ is square size of wire's cross-section, $P_{p}$ is specific weight of wire in case of ice-up, $C_{L 0}, C_{D 0}$ and $C_{M 0}$ are constant aerodynamical coefficients (lifting force, ram pressure and momentum), $\mathrm{V}$ is wind flow speed, $\omega_{A}$ is frequency of linearized system cross vibrations, $\delta_{\Lambda}$ and $\delta_{k}$ are value of linear and torsional motion decrement (during the evaluations were taken as $\delta_{\Lambda} \approx \delta_{k}=\delta$ )

If we denote initial angular coordinate of one of the wires, relatively considered to be the first, like $\mu_{1}$, then angles of $\mu_{i}$ are determined using the formula

$$
\mu_{i}=\mu_{1}+\frac{2 \pi(i-1)}{n} ; i=1 \div n
$$

If distance between neighboring wires in loom $h_{\Pi}$ is known (usually equals to $0.4 \mathrm{~m}$ ), then radius of bundling $R$ determinates as follows

$$
R=\frac{h_{\Pi}}{2 \sin (\pi / n)}=\frac{0,2}{\sin (\pi / n)}
$$

Solution for equation (1) we search in the following form

$$
a(t)=A_{0} e^{\lambda t} \alpha v \delta \varphi(t)=\varphi_{0} e^{\lambda t}
$$

where $A_{0}, \varphi_{0}$ and $\lambda$ are constant, which should be chosen in such way, that system of equation (1) became identities. In order to get nontrivial solution one must chose $\lambda$ in such way, that continuant in the system became zero

$$
\left|\begin{array}{cc}
\lambda^{2}+\left(\frac{\delta \omega_{\Lambda}}{\pi}-k_{1} V\right) \lambda+\omega_{\Lambda}^{2} & k_{7} V^{2} \\
-k_{10} V \lambda & \lambda^{2}+\frac{\delta \omega_{\Lambda}}{\pi} \lambda+\left(s_{1} \omega_{\Lambda}^{2}+k_{10} V^{2}\right)
\end{array}\right|=0
$$

Opening the continuant, we will get characteristic equation of fourth-order

$$
\lambda^{4}+d_{1} \lambda^{3}+d_{2} \lambda^{2}+d_{3} \lambda+d_{4}=0
$$

where

$$
\begin{aligned}
& d_{1}=\left(\frac{2 \delta \omega_{\Lambda}}{\pi}-k_{1} V\right) ; \\
& d_{2}=\left[k_{10} V^{2}-\frac{k_{1} \delta \omega_{\Lambda}}{\pi} V+\omega_{\Lambda}^{2}\left(1+\frac{\delta^{2}}{\pi^{2}}+s_{1}\right)\right] ;
\end{aligned}
$$

$$
\begin{aligned}
& d_{3}=\left[k_{10}\left(k_{7}-k_{1}\right) V^{3}+\frac{k_{10} \delta \omega_{\Lambda}}{\pi} V^{2}-k_{1} s_{1} \omega_{\Lambda}^{2} V+\frac{\delta \omega_{\Lambda}^{3}}{\pi}\left(1+s_{1}\right)\right] \\
& d_{4}=k_{10} \omega_{\Lambda}^{2} V^{2}+s_{1} \omega_{\Lambda}^{4}
\end{aligned}
$$

As is known, from the sign of real component of characteristic marker $\lambda$ depends the character of the researched motion. As task description is in determination of fact of stability or system instability (without pre-computation of characteristic equation root), then for the analysis the Hurwitz criterion may be used.

\section{Main Hurwitz continuant}

$$
\Delta_{4}=\left|\begin{array}{llll}
d_{1} & d_{3} & 0 & 0 \\
1 & d_{2} & d_{4} & 0 \\
0 & d_{1} & d_{3} & 0 \\
0 & 1 & d_{2} & d_{4}
\end{array}\right|
$$

According to Hurwitz, in order to make all polynomial roots (3) have negative actual parts, it is sufficient to make all diagonal minors $\Delta_{4}$ positive, i.e.

$$
\begin{gathered}
\Delta_{1}=d_{1}>0 ; \quad \Delta_{2}=\left|\begin{array}{cc}
d_{1} & d_{3} \\
1 & d_{2}
\end{array}\right|>0 ; \\
\Delta_{3}=\left|\begin{array}{ccc}
d_{1} & d_{3} & 0 \\
1 & d_{2} & d_{4} \\
0 & d_{1} & d_{3}
\end{array}\right|>0 ; \quad \Delta_{4}=d_{4} \Delta_{3}>0 ;
\end{gathered}
$$

Critical speed

Substituting $d_{1}=0$ into $\Delta_{1}=d_{1}>0$ condition, we find the value of critical wind speed

$$
V_{C R 1}=\frac{2 \delta \omega_{\Lambda}}{\pi k_{1}}=\frac{2 P_{P} \delta \omega_{\Lambda}}{2 g \rho d_{\Gamma}\left(C_{D 0}+C_{L 0}\right)}
$$

Next value of critical wind speed may be found from condition

$$
\Delta_{2}=d_{1} d_{2}-d_{3}=0
$$

Substituting the numerical values, we get cubic equation with respect to wind speed

$$
V^{3}+q_{1} V^{2}+q_{2} V+q_{3}=0
$$

where

$$
q_{1}=-\frac{\delta \omega_{\Lambda}}{\pi k_{7} k_{10}}\left(k_{1}^{2}+k_{10}\right) ; q_{2}=\frac{k_{1} \omega_{\Lambda}^{2}}{k_{7} k_{10}} ; q_{3}=-\frac{\left(1+s_{1}\right) \delta \omega_{\Lambda}^{3}}{\pi k_{7} k_{10}} ;
$$

To solve the equations we may use iterative method of characteristic equation roots determination [17-19]. Computation using this method is done using several step-by- 
step approaches. Process of computation converges quickly enough. As a result of using this method it is possible to decompose equation of third-order as follows (Assuming the smallest root by modulus to be actual)

$$
(V+\alpha)\left[V^{2}+\left(q_{1}-\alpha\right) V+\left(q_{2}-\beta\right)\right]=0
$$

where $\alpha=\frac{q_{3}}{q_{2}-\beta} ; \beta=\alpha\left(q_{1}-\alpha\right)$;

Initial (first) approach is determined when $\beta=0$.

$$
\alpha=\frac{q_{3}}{q_{2}}=-\frac{\left(1+s_{1}\right) \delta \omega_{\Lambda}}{\pi k_{1}} ;
$$

Substituting to equation (7), we find lower border of critical speed value in first approach

$$
V_{C R 2}=\frac{\left(1+s_{1}\right) \delta \omega_{\Lambda}}{\pi k_{1}}=\frac{\left(1+s_{1}\right) P_{P} \delta \omega_{\Lambda}}{2 g \rho d_{\Gamma}\left(C_{D 0}+C_{L 0}\right)}
$$

Roots of the second bracket in the equation (7) are compound and linked.

The second approach doesn't give any serious corrections; therefore, further we will take initial approaches.

From condition

$$
\Delta_{3}=d_{1} d_{2} d_{3}-d_{1}^{2} d_{4}-d_{3}^{2}=0
$$

we get the following equation for critical speed of wind flow (during transformation process we have ignored the equation terms, which don't have any significant effect on final result)

$$
V^{6}+q_{1} V^{5}+q_{2} V^{4}+q_{3} V^{3}+q_{4} V^{2}+q_{5} V+q_{6}=0
$$

where

$$
\begin{aligned}
& q_{1}=-\frac{k_{1} \delta \omega_{\Lambda}}{\pi k_{7}\left(k_{1}-k_{7}\right)}\left[1+\frac{2 k_{1}\left(k_{1}-k_{7}\right)}{k_{10}}\right] ; q_{2}=-\frac{k_{1}\left(1-s_{1}\right) \omega_{\Lambda}^{2}}{k_{10}\left(k_{1}-k_{7}\right)} ; \\
& q_{3}=-\frac{k_{1}^{3} s_{1} \delta \omega_{\Lambda}^{3}}{\pi k_{7} k_{10}^{2}\left(k_{1}-k_{7}\right)}\left[1-\frac{2 k_{10}\left(1-s_{1}\right)}{k_{1}^{2} s_{1}}\right] ; \\
& q_{4}=-\frac{2\left(1+s_{1}\right) \delta^{2} \omega_{\Lambda}^{4}}{\pi^{2} k_{7} k_{10}\left(k_{1}-k_{7}\right)} ; \\
& q_{5}=-\frac{k_{1}\left(1-s_{1}\right)^{2} \delta \omega_{\Lambda}^{5}}{\pi k_{7} k_{10}^{2}\left(k_{1}-k_{7}\right)} ; \\
& q_{6}=\frac{\left(1+s_{1}\right)^{2} \delta^{2} \omega_{\Lambda}^{6}}{\pi^{2} k_{7} k_{10}^{2}\left(k_{1}-k_{7}\right)}
\end{aligned}
$$

Similarly, decomposing the left-hand side of equation (10) to multipliers, we get

$$
\begin{aligned}
& (V+\alpha)\left[V^{5}+\left(q_{1}-\alpha\right) V^{4}+\left(q_{2}-\beta\right) V^{3}+\right. \\
& \left.+\left(q_{3}-\gamma\right) V^{2}+\left(q_{4}-\psi\right) V+\left(q_{5}-\theta\right)\right]>0
\end{aligned}
$$

where values $\alpha ; \beta ; \gamma ; \psi ; \theta$ are determined according to expressions

$$
\begin{aligned}
& \alpha=\frac{q_{6}}{q_{5}-\theta} ; \beta=\alpha\left(q_{1}-\alpha\right) ; \gamma=\alpha\left(q_{2}-\beta\right) ; \\
& \psi=\alpha\left(q_{3}-\gamma\right) ; \theta=\alpha\left(q_{4}-\psi\right) ;
\end{aligned}
$$

First approach determines when $\theta=0$.

$$
\alpha=\frac{q_{6}}{q_{5}}=-\frac{\left(1+s_{1}\right)^{2} \delta \omega_{\Lambda}}{\pi k_{1}\left(1-s_{1}\right)^{2}}
$$

From the first bracket of the equation (11) we find value of critical speed in first approach

$$
V_{C R 3}=\frac{\left(1+s_{1}\right)^{2} \delta \omega_{\Lambda}}{\pi k_{1}\left(1-s_{1}\right)^{2}}=\frac{\left(1+s_{1}\right)^{2} P_{P} \delta \omega_{\Lambda}}{2 g\left(1-s_{1}\right)^{2} \rho d_{\Gamma}\left(C_{D 0}+C_{L 0}\right)}
$$

The second bracket of equation (11) gives two roots with negative sign, one pair of compound roots and one root is positive. If root is positive, wind speed modulus equals to more than $40 \mathrm{~m} / \mathrm{s}$, so vibrations of the bundled phase are nearly impossible. That's why, the second bracket of the equation (11) we exclude from the analysis.

Condition $\Delta_{4}=d_{4} \Delta_{3}>0$ is true when $\Delta_{3}>0$, because coefficient $d_{4}>0$ when wind speed may be different.

Critical wind speeds, which determine borders of the bundled phase stability, are placed in increasing order as follows

$$
V_{C R 2}<V_{C R 1}<V_{C R 3}
$$

Note, that from all of the Hurwitz's conditions, the most rigid condition is $\Delta_{2}>0$, which leads to critical speed

$$
V_{C R 2}=\frac{\left(1+s_{1}\right) P_{P} \delta \omega_{\Lambda}}{2 g \rho d_{\Gamma}\left(C_{D 0}+C_{L 0}\right)}=\frac{\pi\left(1+s_{1}\right) P_{P} \delta}{2 g \rho d_{\Gamma} \ell\left(C_{D 0}+C_{L 0}\right)} \sqrt{\frac{g T}{P_{P}}\left(1+\frac{8 E F P_{P}^{2} \ell^{2}}{\pi^{4} T^{3}}\right)}
$$

If $V \geq V_{C R 2}$, then stability of bundled phase is violated, i.e. vibration is increasing over time.

As it is seen from formula (14), critical speed depends on characteristics of line itself and aerodynamical characteristics of precipitations. As example, Figure II shows the change of critical galloping speed of the bundled phase in accordance to various characteristics. Computation is done for AC-300/39 wire with the following characteristics: $E=7700 \mathrm{daN} / \mathrm{mm}^{2} ; \quad F$ $=339,6 \mathrm{~mm}^{2}$; let specific resulting load on wire be equal to $P_{P} \approx 1,32 \mathrm{daN} / \mathrm{m}$;

For $n=2 ; R=0,2 \mathrm{~m}$ and $s_{1}=1$. 
For $n=3 ; R=0,23 \mathrm{~m}$ and $s_{1}=0,5$.

Results of computation are shown in Figures II $-\mathrm{V}$.

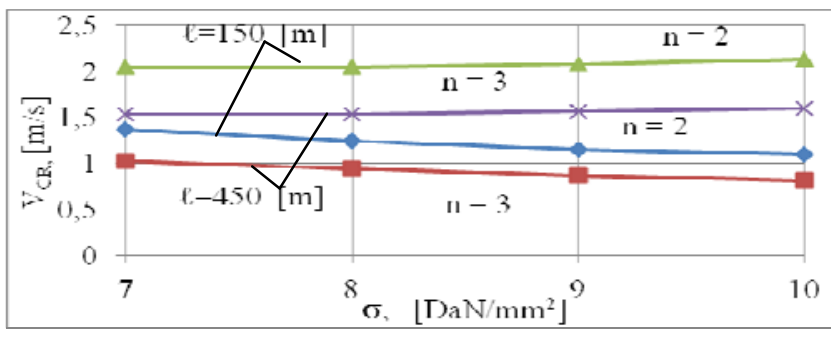

FIGURE II. DEPENDENCE OF CRITICAL SPEED ON MECHANICAL STRESS OF WIRE. COMPUTATIONS ARE DONE WITH: $P=0,12$ DAN C $/ \mathrm{M}^{4}$.

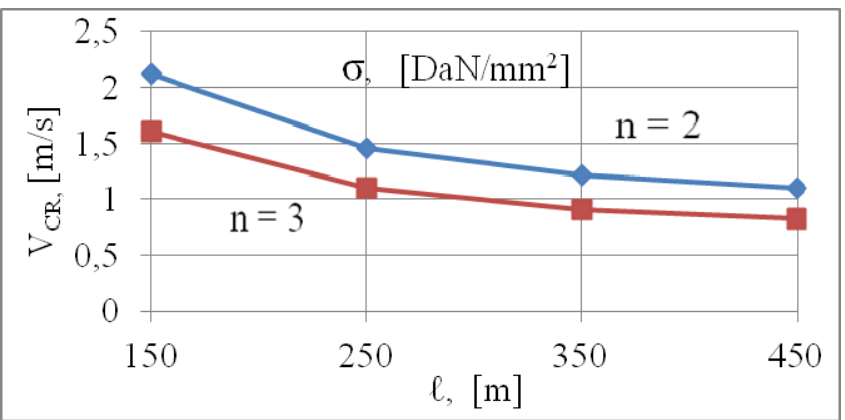

FIGURE III. DEPENDENCE OF CRITICAL SPEED ON SPAN LENGTH. COMPUTATIONS ARE DONE WITH: $P=0,12$ DAN C ${ }^{2} / \mathrm{M}^{4} ; B_{\Gamma}=0$, $04 \mathrm{M} ; \Delta=0,1 ; C_{D 0}=0,87 ; C_{L 0}=4 ; \Sigma=10\left[\mathrm{DAN} / \mathrm{MM}^{2}\right]$

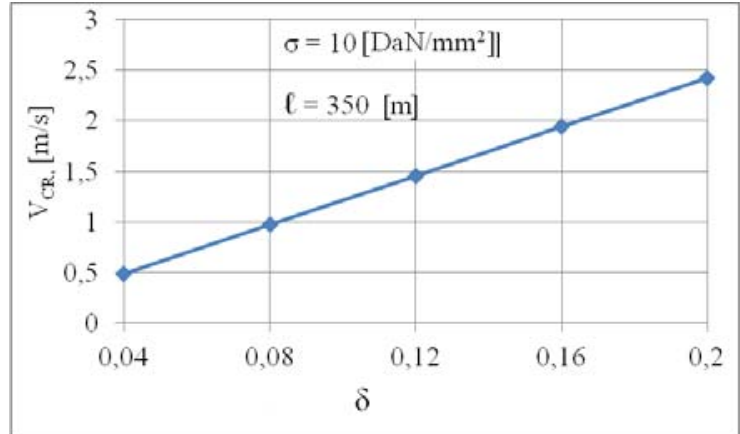

FIGURE IV. DEPENDENCE OF CRITICAL SPEED ON ATTENUATION RATIO. COMPUTATIONS ARE DONE WITH: $P=0,12 \mathrm{DAN} \mathrm{C}^{2} / \mathrm{M}^{4}$ $B_{\Gamma}=0,04 \mathrm{M} ; \mathrm{N}=2$.

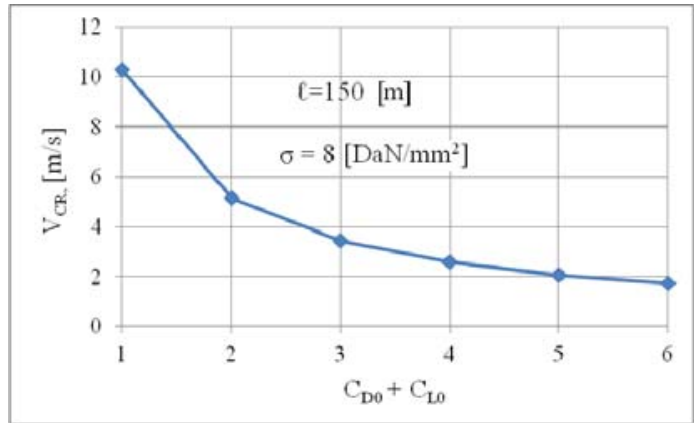

FIGURE V. DEPENDENCE OF CRITICAL SPEED ON AERODYNAMICAL COEFFICIENTS. COMPUTATIONS ARE DONE WITH: $P=0,12$ DAN C ${ }^{2} / \mathrm{M}^{4} ; B_{\Gamma}=0,04 \mathrm{M}$; AND N $=2$.

\section{CONCLUSIONS}

1) All executed computations show (Figures II and III), that theoretically the bundled phase, consisted of two wires, is more stable to appearance of galloping in comparison with the bundled phase, consisted of three wires. Practically, they exposed to galloping (in the same conditions) equally, because differences of critical speed modulus for these structures the bundled phase are negligibly small.

2) Increasing the length of span decreases the critical wind speed value, consequently, increasing the length of span decreases the bundled phase aerodynamical stability.

3) Influence of mechanical stress on critical speed value is ambiguous. For small span lengths, increase of voltage comes amid increasing of critical speed modulus, and if one increase length of span, then this mechanism works vice versa. This is connected to complex relationship between vibration frequency, span length and mechanical stress of wire.

4) Increasing vibrations decrement the critical speed modulus increasing linearly. This circumstance may be used in the design of wires galloping control methods.

5) In order to increase aerodynamical stability the bundled phase, it is necessary to decrease coefficient value of aerodynamical characteristics of ice precipitation. This might be reached, for example, by setting such absorbers, which aerodynamical characteristics are opposite by sign to aerodynamical characteristics of ice precipitation.

6) Linear statement of aim allows setting conditions of vibration process appearance, but doesn't give opportunities for prediction of further vibration development, because non-linear components of vibration process are not taken into consideration. Nevertheless, it gives opportunity for qualitative estimation of influence degree of one or another characteristic on critical wind flow speed, thus, on aerodynamical stability of bundled phase.

\section{REFERENCES}

[1] Den Hartog J.P., Transmission line Vibration due to Sleet. Trans AIEE. 1930; 49

[2] Lilien J, Havard D. Galloping data base on single and bundle conductors prediction of maximum amplitudes. Prediction of maximum amplitudes. IEEE Trans on Power Delivery 2000;15(2):670-674.

[3] Richardson A. A study of galloping conductors on a $230 \mathrm{kV}$ transmission line. Electric Power Systems Research 1991; 21(1):43-55.

[4] Xiao-hui LIU, Bo YAN, Hong-yan ZHANG, Song ZHOU. Nonlinear numerical simulation method for galloping of iced conductor. Applied Mathematics and Mechanics 2009; 30(4):489-501.

[5] Nigol, O. and Buchan, P. G., Conductor Galloping 2: Torsional Mechanism. IEEE Transactions on Power Apparatus and Systems. 1981; 100(2): 708-720

[6] Muhammad Bilal Waris, Takashi Ishihara, Muhammad Waheed Sarwar Galloping response prediction of ice-accreted transmission lines. The 4th International conference on Advances in Wind and Structures (AWAS'08) Jeju, Korea, May 29-31, 2008.

[7] Nakamura Y. Galloping of bundled power line conductors. Journal of Sound and Vibration. 1980; 73(3): 363-377.

[8] Zhang Q., Popplewell N., Shah A. H. Galloping of bundle conductor. Journal of Sound and Vibration. 2000; 234(1):115-134. 
[9] Xijun Liu, Xiaoqin Sun, Suxia Zhang, Peng Liu, Bing Liu. Comparative analysis of dynamic characteristics under two iced conductor galloping models. Applied Mechanics and Materials. 2011; 94-96:1685-168

[10] Xin Wang, Wen-Juan Lou. Numerical approach to galloping of conductor. The Seventh Asia-Pacific Conference on Wind Engineering. November, 2009.

[11] Zhao Li, Yan Bo, Cai Meng-qi, Wu Chuan. Numerical investigation on galloping characteristics of continuous multi-span iced quad bundle conductor lines. Applied Mathematics and Mechanics. 2014; 35(5):487497.

[12] Mohamed Abdel-Rohman, Ayman Mugahed. Using tuned mass dampers to suppress galloping of tall cantilever structures: A two dimensional analysis. Kuwait J. Sci. Eng. 2008; 35(2B):55-78.

[13] LI Xin-ye, ZHANG Hua-biao, HOU Shu-jun, GAO Shi-zhao. Theoretical and numerical analysis of galloping of iced power transmission lines. Journal of Vibration Engineering. 2010; 23(1)

[14] John D. Holmes. Wind Loading of Structures, Third Edition. 2015

[15] Li Xinmin, Zhu Kuanjun, Liu Bin, Research of Experimental Simulation on Aerodynamic Character for Typed Iced Conductor, AASRI Conference on Power and Energy Systems. 2 (2012) 106-111.

[16] J. Y. Cai, X. J. Liu, S. X. Zhang. Numerical Analysis for Galloping of Iced Quad Bundle Conductors. Applied Mechanics and Materials. 2012; 226-228: 30-34

[17] Tokenov N., Dzhamanbayev M., Bekbayev A., Eskendirova D., Baimuratov O. Mathematical Model for Calculating Aerodynamic Characteristics of Overhead Transmission Lines. Applied Mechanics and Materials. 2014; 610:52-59.

[18] Fedor N.SHKLYARCHUK, Alexander N. DANILIN, Jean-Louis LILIEN, Dmitry V. SNEGOVSKIY, Alexander A. VINOGRADOV, Murat A. DJAMANBAYEV Nonlinear aero-elastic vibrations and galloping of iced conductor lines under wind.- Seventh International Symposium CABLE DYNAMICS Vienna (Austria), December 10-13, 2007.

[19] Djamanbaev M.A., Tokenov N. «Mathematical model of aerial overhead lines bundled phase galloping (Anchor span)» Of the II - international scientific conference "High tech means sustainable development", Almaty, 23-24, may, 2013y. 